\title{
A note on the effects of local blockage and dynamic tuning on tidal turbine performance
}

\author{
Lei Chen \\ Department of Engineering Science \\ University of Oxford \\ Oxford OX1 3PJ, UK \\ e-mail: lei.chen@eng.ox.ac.uk \\ Paul A. J. Bonar \\ School of Engineering \\ University of Edinburgh \\ Edinburgh EH9 3FB, UK \\ e-mail: p.bonar@ed.ac.uk
Christopher R. Vogel
Department of Engineering Science
University of Oxford
Oxford OX1 3PJ, UK
e-mail: christopher.vogel@eng.ox.ac.uk \\ Thomas A. A. Adcock \\ Department of Engineering Science \\ University of Oxford \\ Oxford OX1 3PJ, UK \\ e-mail: thomas.adcock@eng.ox.ac.uk
}

Numerical simulations are used to explore the potential for local blockage effects and dynamic tuning strategies to enhance the performance of turbines in tidal channels. Fulland partial-width arrays of turbines, modelled using the volume-flux-constrained actuator disc and blade element momentum theories, are embedded within a two-dimensional channel with a naturally low ratio of drag to inertial forces. For steady flow, the local blockage effect observed by varying the cross-stream spacing between the turbines is found to agree very well with the predictions of the two-scale actuator disc theory of Nishino and Willden (2012, "The efficiency of an array of tidal turbines partially blocking a wide channel”, J. Fluid Mech., vol. 708, pp. 596-606). For oscillatory flow, however, results show that, consistent with the findings of Bonar et al. (2019, "On the arrangement of tidal turbines in rough and oscillatory channel flow", J. Fluid Mech., vol. 865, pp. 790-810), the shorter and more highly blocked arrays produce considerably more power than predicted by two-scale theory. Results also show that, consistent with the findings of Vennell (2016, "An optimal tuning strategy for tidal turbines”, Proc. R. Soc. A., vol. 472, p. 20160047), the 'dynamic' tuning strategy, in which the tuning of the turbines is varied over the tidal cycle, can only produce significantly more power than a temporally fixed turbine tuning if the array has a large number of turbine rows or a large local blockage ratio. For all cases considered, trends are consistent between the two turbine representations but the effects of local blockage and dynamic tuning are found to be much less significant for the more realistic tidal rotor than for the idealised actuator disc.

\section{Introduction}

The task of maximising the power produced by a tidal turbine array is complicated by the need to optimise the position and operation of each turbine with respect to those of all other turbines [1]. However, idealised theoretical models can be used to simplify this complex optimisation problem and provide valuable insights to inform array design.

Garrett and Cummins [3], for instance, used an actuator disc model to show that for a single turbine (or fullwidth turbine array) operating in volume-flux-constrained 
(a)

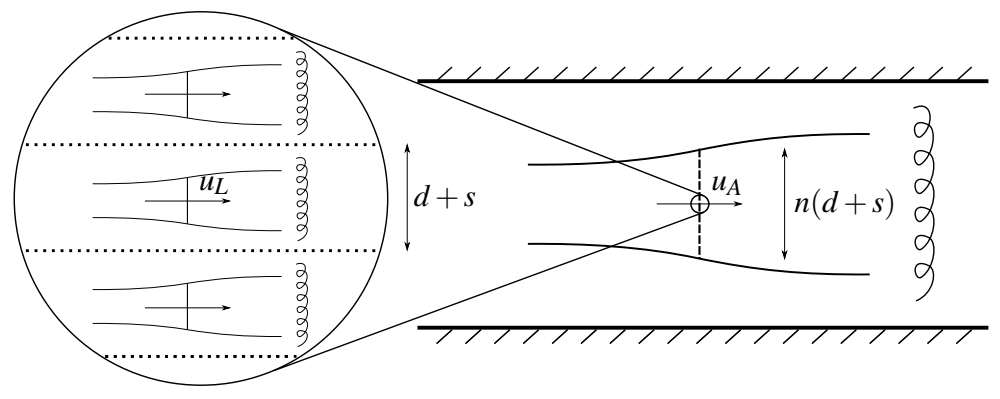

(b)

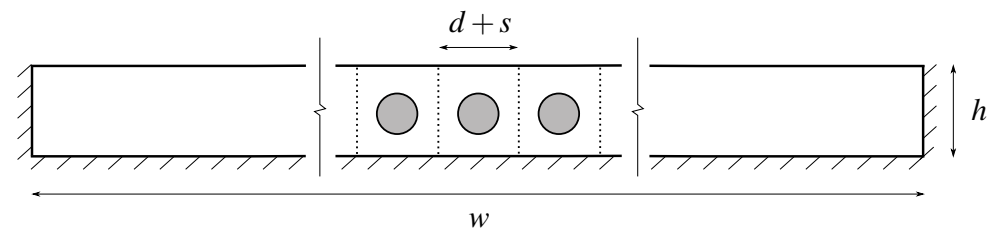

Fig. 1: Schematic representation of the two-scale actuator disc model for volume-flux-constrained flow, showing: $(a)$ plan views of the local- (left) and array-scale (right) flows, and; $(b)$ streamwise cross-sectional view of the partial-width tidal turbine array. Adapted from Nishino and Willden [2].

flow (meaning flow with a 'rigid lid' which does not deform in response to energy extraction), the maximum power depends on the global blockage ratio, which is defined as the ratio of turbine (or array) swept area to flow cross-sectional area. It is for this reason that the effects of blockage must be accounted for both in the design of tidal turbines [4] and when comparing performance characteristics between devices operating under different blockage ratios [5]. Nishino and Willden [2] and Vogel et al. [6] later extended this actuator disc model to examine the maximum power that can be produced by tidal turbine arrays spanning only part of the flow cross-section. By assuming that array-scale flow events take place over much greater time and length scales than local-scale flow events, these authors were able to describe the performance of a partial-width array using two loosely coupled actuator disc models to describe the localand array-scale flows (Fig. 1). They then showed that for each global blockage, which is now defined as the product of a local blockage (the ratio of turbine frontal area to local flow passage cross-sectional area) and an array blockage (the ratio between the cross-sectional areas of the array and channel), there exists an optimal local blockage (or, equally, an optimal array blockage) which produces a peak array power output. This optimal local blockage can be achieved by optimising the cross-stream spacing between the turbines $[2,6]$.

The two-scale actuator disc theory of Nishino and Willden [2] can be used to predict the optimal arrangement of, and maximum power available to, tidal turbines arranged in a single cross-stream row. However, these predictions will be limited by the simplifying assumptions underlying the theory, which include the assumption that the mass flux through the channel is unaffected by the resistance presented by the turbines. To account for the reduction in channel-scale flow, the two-scale theory must first be coupled with a model of channel-scale dynamics. The simplest such model is that due to Garrett and Cummins [7], which describes the interactions between the resistance presented by the array and the bulk flow through the channel. However, a recent paper by Bonar et al. [8] has shown that under certain oscillatory flow conditions, this coupled two-scale theory is unable to predict the optimal arrangement of, and considerably underpredicts the maximum power available to, turbines operating in a two-dimensional tidal channel. Using a numerical model of actuator discs in shallow water, Bonar et al. [8] identified new two-dimensional dynamics that develop around a partial-width array in inertial oscillatory flow which are not captured by the one-dimensional two-scale theory. In such cases, Bonar et al. [8] found that grouping the turbines close together so as to maximise the effects of these new arrayscale dynamics produced considerably more power than predicted by two-scale theory.

In this paper, we use a similar numerical model to extend the work of Bonar et al. [8] to consider multiple rows of turbines, dynamic turbine tuning strategies, and a more realistic turbine representation, which is based on the volumeflux-constrained blade element momentum theory of Vogel et al. [9]. Actuator disc theory has been used extensively in analytical and numerical models of tidal stream power, and has been used to calculate upper bounds on the power available to turbines placed at candidate sites [10-12], but the uniformly porous disc provides only an idealised description of turbine performance. By contrast, the more advanced blade element momentum rotor is able to better describe the forces developed and power produced by real turbine blades.

In the first part of the paper, the two idealised models of axial flow turbines are embedded within two different channel domains: the one-dimensional analytical channel of Garrett and Cummins [7], and a two-dimensional numerical channel, following Bonar et al. [8]. Different turbine arrangements are then considered for both steady and oscillatory flow conditions, and turbine performance is compared between the different channel and turbine representations in terms of both the maximum time-averaged available power, which is obtained by optimising the temporally fixed turbine 
tuning, and the maximum global power coefficient, which is defined here as the ratio of maximum time-averaged available power to the time- and cross-stream-averaged channelscale kinetic energy flux. This part extends the work of Bonar et al. [8] to include multiple turbine rows and a more realistic turbine model. The results are then used to provide a better understanding of the effects of local blockage on tidal turbine performance, and further assess the potential for existing theories to capture these effects.

In the second part of the paper, numerical simulations are used to explore the potential for dynamic tuning strategies, such as those proposed by Vennell and Adcock [13] and Vennell [14], to enhance the power available to rows of turbines spanning only part of the channel cross-section. As before, different turbine arrangements are considered and the maximum time-averaged available power is compared between the different turbine models. This part extends not only the work of Bonar et al. [8] to include dynamic tuning, but also the work of Chen et al. [15] to include partial-width turbine arrays. Although the computational demands of the numerical scheme used to calculate the optimal tuning curves limit the study to a single channel and small number of turbine arrangements, the present paper is, to our knowledge, the first to explore the combined effects of local blockage and dynamic tuning on tidal turbine performance.

\section{Models}

\subsection{Actuator disc}

Actuator disc theory provides the simplest representation of an axial flow turbine, modelling the rotor plane as a porous disc presenting a uniform resistance to the flow passing through. The classical unbounded disc model has been extended to volume-flux-constrained flow by Garrett and Cummins [3], and then further extended by other authors to investigate more realistic flow conditions and optimal turbine arrangements [2, 6, 16-21].

In actuator disc theory, turbine performance is defined by three dimensionless parameters: the blockage ratio, $B$, which represents the ratio of turbine swept area to flow crosssectional area; the basin efficiency, $\eta$, which represents, to leading order, the ratio between the power and thrust coefficients, $C_{P}$ and $C_{T}$; and the tuning parameter, which is typically represented by a wake velocity coefficient, $\alpha_{4}[16,17]$. Alternatively, the tuning parameter can be expressed as a resistance coefficient, $k$, given by $k=\left(\beta_{4}^{2}-\alpha_{4}^{2}\right) / \alpha_{2}^{2}$, in which $\alpha_{2}\left(=1-a_{2}\right), \alpha_{4}\left(=1-a_{4}\right)$, and $\beta_{4}$ are the throughflow, wake, and wake bypass velocity coefficients [16,17]; $a_{2}$ and $a_{4}$ are the throughflow and wake induction factors [9]; and the subscripts ' 2 ' and ' 4 ' are used to refer, respectively, to the location immediately upstream of the turbine and the point of cross-stream pressure equalisation in its near wake $[16,17]$. Whilst the more commonly used velocity coefficients are defined with reference to a velocity upstream of the disc, the resistance coefficient is a measure of local thrust based on the throughflow velocity $[19,20]$. As such, the resistance coefficient offers a more intuitive description of disc porosity, which can be more easily related to the geometric properties of the physical porous discs often used in laboratory experiments [19-22].

\subsection{Blade element momentum rotor}

Blade element momentum theory provides a more advanced low-order turbine representation, combining linear and rotational momentum theory with blade element theory to better describe the forces developed and power produced by real turbine blades. The classical blade element momentum theory was derived to analyse the performance of wind turbines, and so does not account for the blockage effects which contribute significantly to tidal turbine performance [3, 4, 16, 17, 22, 23]. However, Vogel et al. [9] have recently extended this model to volume-flux-constrained flow in order to analyse the performance of tidal turbines. This blockage-corrected theory accounts for the development of a streamwise static pressure difference which enables the rotor to apply a greater $C_{T}$ and thereby achieve a higher $C_{P}$. This tidal rotor model has also been compared favourably with blade-resolved computational fluid dynamics simulations. Further details of the model derivation and validation are provided by Vogel et al. [9, 24].

As with the actuator disc, the key variables for the tidal rotor are $B, \eta$, and the tuning parameter. In this case, however, the wake velocity coefficients are replaced by variables more closely related to the operation of the rotor: the blade pitch angle, $\beta$, and the tip speed ratio, $T S R$, which defines the ratio of the tangential speed of the blade tip (the product of the angular velocity and rotor radius) to the throughflow velocity. The resistance coefficient, $k$, may also be expressed in terms of $T S R$ and $\beta$ to facilitate a direct comparison between rotor and disc performance. Although the rotor provides a more accurate description of turbine performance than the disc, it retains some limitations that restrict its ability to model large blockage ratios and extreme off-design conditions. For large $T S R$, for instance, the rotor attains a high throughflow induction factor and the model begins to produce non-physical solutions as the wake flow becomes turbulent [15]. In the present study, such solutions are precluded by limiting $B$ to values $\leq 0.3125$ and $T S R$ to a realistic range of operation: $3 \leq T S R \leq 8.5$. Though not considered here, the operational range of $T S R$ is likely to be further restricted, particularly for large blockage ratios, so as to avoid blade cavitation [25].

It is also worth noting that although the present study employs both temporally fixed and dynamic turbine tuning strategies, array performance is measured only in terms of the time-averaged available power and global power coefficient. Moreover, although rotor performance is defined by a realistic performance curve, this study does not examine in detail, as Vogel et al. [26] have, the change in performance characteristics above rated power or the effects of power capping on the dynamics of flow through the channel. 

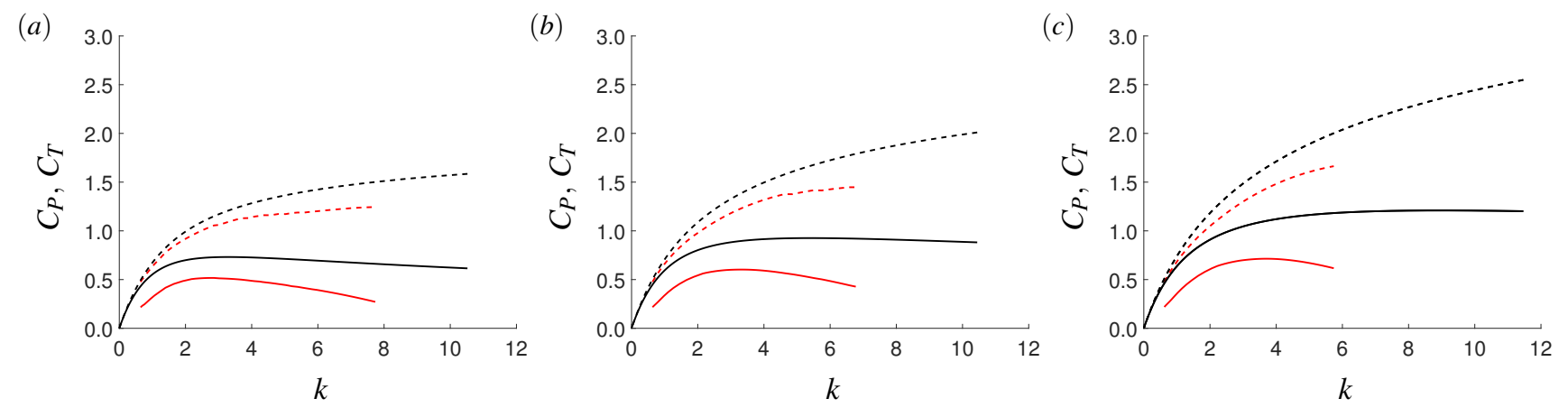

Fig. 2: Variations in power coefficient, $C_{P}$ (solid lines), and thrust coefficient, $C_{T}$ (dashed lines), with resistance coefficient, $k$, for an actuator disc (black) and tidal rotor (red) operating with: $(a)$ low $(B=0.1) ;(b)$ moderate $(B=0.2)$, and; $(c)$ high $(B=0.3)$ blockage ratios. (Colour online.)
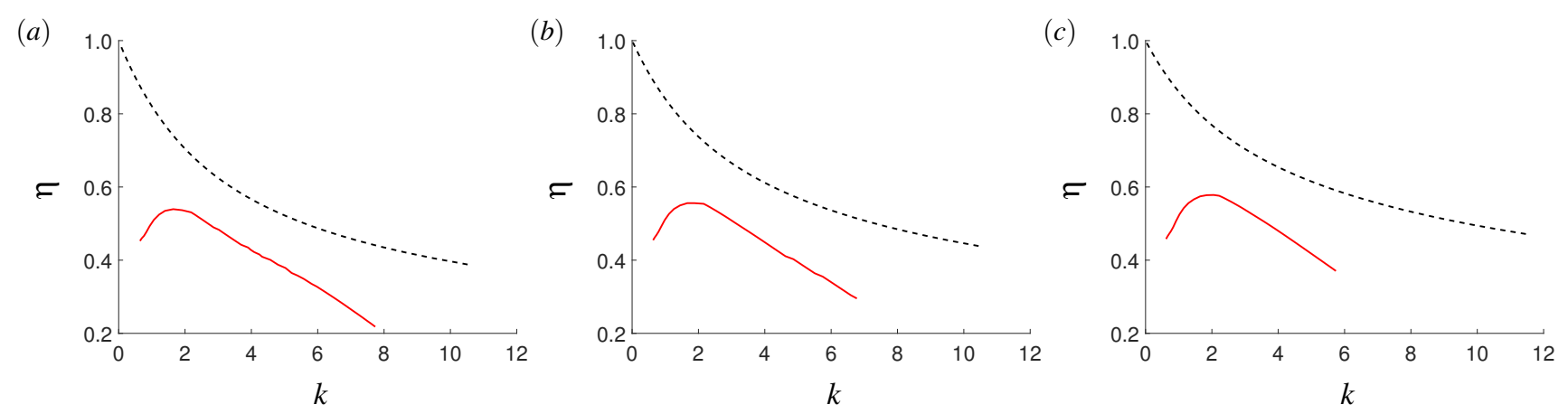

Fig. 3: Variations in basin efficiency, $\eta$, with resistance coefficient, $k$, for an actuator disc (dashed black lines) and tidal rotor (solid red lines) operating with: $(a)$ low $(B=0.1) ;(b)$ moderate $(B=0.2)$, and; $(c)$ high $(B=0.3)$ blockage ratios. $($ Colour online.)

\subsection{Disc and rotor performance comparisons}

Schluntz et al. [4] have demonstrated the importance of designing turbines for a specific blockage ratio. Here, a single fixed-pitch rotor with a diameter of $20 \mathrm{~m}$ is used for convenience. Following Cao et al. [27], this rotor is designed for an inflow velocity $u_{A}=2 \mathrm{~m} / \mathrm{s}, B=0.16$, and $T S R=5.5$, and the blades are based on the Ris $\emptyset-\mathrm{A} 1-24$ hydrofoil, which has been found to provide good agreement between the tidal rotor model and blade-resolved computational fluid dynamics simulations [27, 28].

The differences in performance between this rotor and the equivalent actuator disc are most clearly shown in Figs 2 and 3, which demonstrate, for each model, how $C_{P}, C_{T}$, and $\eta$ vary with $k$ under three different off-design blockage ratios: $B=0.1,0.2$, and 0.3 . Table 1 shows the maximum power coefficients, $C_{P(\max )}$, and corresponding optimal thrust and resistance coefficients, $C_{T(o p t)}$ and $k_{(o p t)}$, and basin efficiencies, $\eta_{(o p t)}$. Although the variations in $C_{P}$ and $C_{T}$ are broadly similar, Fig. 2 shows that the magnitudes of $C_{P}$ and $C_{T}$ are lower for the more realistic rotor, which experiences both lift and drag forces, than for the idealised disc [15]. Figure 3 shows that the variations in $\eta$ are also quite different. The efficiency of the rotor, which experiences additional energy losses, such as blade tip losses, is much lower than that of the disc, which is assumed to lose energy only to wake mixing. Moreover, for the disc, there is a simple inverse relationship between $k$ and $\eta$ : as $k$ increases, so does $C_{T}$ and
Table 1: Maximum power coefficient, $C_{P(\max )}$, and corresponding optimal thrust and resistance coefficients, $C_{T(o p t)}$ and $k_{(o p t)}$, and basin efficiency, $\eta_{(o p t)}$, for tidal rotors and actuator discs operating with blockage ratios, $B$

\begin{tabular}{cccccc}
\hline$B$ & Turbine & $C_{P(\max )}$ & $C_{T(\text { opt })}$ & $k_{(\text {opt })}$ & $\eta_{(\text {opt })}$ \\
\hline 0.1 & Rotor & 0.51 & 1.05 & 2.85 & 0.50 \\
0.1 & Disc & 0.73 & 1.21 & 3.29 & 0.61 \\
\hline 0.2 & Rotor & 0.60 & 1.23 & 3.37 & 0.49 \\
0.2 & Disc & 0.93 & 1.67 & 5.40 & 0.55 \\
\hline 0.3 & Rotor & 0.71 & 1.43 & 3.76 & 0.49 \\
0.3 & Disc & 1.21 & 2.39 & 8.96 & 0.51 \\
\hline
\end{tabular}

thus the amount of power dissipated in wake mixing, so $\eta$ decreases. For the fixed-pitch rotor, however, the relationship is more complex: as $k$ increases from zero, $\eta$ initially increases as the TSR increases and flow begins to attach to the surfaces of the blades, peaks at the design TSR, and then decreases as the $T S R$ continues to increase and drag forces begin to predominate [15]. 


\subsection{One-dimensional analytical channel}

Garrett and Cummins [7] proposed a theoretical model to describe the extraction of tidal stream energy from a simple tidal channel. In this one-dimensional theory, the tidal forcing is assumed to be unaffected by changes in the channel flow rate, and the turbine array, which is represented as a simple opposing force, is assumed to span completely the channel width. Vennell [18] later combined this model with the volume-flux-constrained actuator disc theory to analyse the performance of turbine arrays spanning tidal channels.

The work of Vennell [18] can be extended to include partial-width arrays by replacing the volume-fluxconstrained actuator disc model of Garrett and Cummins [3] with the two-scale actuator disc model of Nishino and Willden [2]. In two-scale theory, the local blockage ratio, $B_{L}$, is defined as the ratio of turbine frontal area, $\pi d^{2} / 4$, where $d$ is the diameter of each of the $n$ identical turbines, to the cross-sectional area of the local flow passage, $h(d+s)$, where $h$ is the water depth and $s$ is the cross-stream inter-turbine spacing (Fig. 1). The global blockage ratio is then defined as $B_{G}=B_{A} B_{L}\left(=n \pi d^{2} / 4 h w\right)$, where $w$ is the channel width and $B_{A}$ is the array blockage, which represents the ratio between the cross-sectional areas of the array, $h n(d+s)$, and the channel, $h w$. Willden et al. [29] and Gong et al. [30] have also sought to combine two-scale theory with the simple channel model of Garrett and Cummins [7] in order to analyse the performance of partial-width arrays in channels. More recently, however, Bonar et al. [8] have shown that these coupled one-dimensional models may, under certain oscillatory flow conditions, neglect leading-order physics.

\subsection{Two-dimensional numerical channel}

Following Bonar et al. [8], this study also employs a two-dimensional channel model, which uses the discontinuous Galerkin (DG) version of the open-source hydrodynamic model ADCIRC to solve the depth-averaged shallow water equations [31,32]. Following Draper et al. [33], the turbines are embedded within the model at sub-grid scale, and the extraction of energy is represented as a discontinuous reduction in fluid depth, with local-scale mixing simulated analytically and array-scale mixing simulated numerically by means of a spatially and temporally constant horizontal eddy viscosity coefficient. Though simplistic, this line sink modelling approach has been widely used in numerical studies of tidal stream power, both in idealised domains $[8,34]$ and at real sites [10-12], and has been found to agree well with laboratory-scale physical experiments [35]. The present numerical code is an updated version of that used by Adcock et al. [10] and other authors, which has been modified by Schnabl et al. [36] to allow the turbine performance to be defined by specified power and thrust curves.

The numerical channel is $30 \mathrm{~km}$ long, $10 \mathrm{~km}$ wide, 30 $\mathrm{m}$ deep, and divided into 2,038 triangular elements which vary in size from $200 \mathrm{~m}$ near the array to $1 \mathrm{~km}$ at the channel boundaries. A time step of $1 \mathrm{~s}$ is specified and results obtained using the present grid are found to agree to within $1 \%$ with those obtained using twice and half as many numer- ical elements. Estimates of undisturbed channel flow rate and power produced by the full-width array are then found to agree to within $1 \%$ with the respective theoretical models $[7,18]$. The channel walls are assigned slip boundary conditions, and a typical bed roughness coefficient of $C_{d}=0.002$ is selected [37]. Steady flow is driven by assigning a constant inflow velocity of $1 \mathrm{~m} / \mathrm{s}$ at the model inlet. This boundary condition is used to facilitate comparison with the two-scale theory, which assumes that the channel flow rate is unaffected by turbine resistance [2]. Oscillatory flow is driven by raising and lowering the water level at the model inlet with amplitude $0.2 \mathrm{~m}$ and period, $T=12.42 \mathrm{~h}$. These values correspond to a natural dynamic balance of $\lambda_{0}=0.2$ [7], which suggests a naturally low ratio of drag to inertial forces and hence that the effects of local blockage and dynamic tuning should be significant $[8,14]$. For each simulation, the analytical and numerical models are allowed to spin up for 2 days (model time) and then run for a further 8 days before the results from the last 2 complete tidal cycles are extracted for analysis.

\subsection{Dynamic turbine tuning strategies}

In analysing the performance of full-width arrays in channels, Vennell [18] adopted a fixed tuning strategy: the turbine wake velocity coefficient was held constant as the throughflow velocity varied over the tidal cycle and tuned to maximise the time-averaged available power. Adcock [38] then showed that a temporally varying turbine tuning can produce considerably more power than a temporally fixed tuning, and Vennell [14] later extended this work to show that a dynamic tuning strategy can yield triple benefits: the turbines can produce more power over the tidal cycle, without increasing the peak structural loads, and whilst also maintaining a higher channel flow rate. Vennell and Adcock [13] have also shown that dynamic tuning can provide a degree of flexibility as to when power is produced, and so can be used to better meet short, predictable peaks in power demand.

The above analytical models used Fourier series and gradient-based optimisation methods, but here a simple 'generate-and-test' approach is used to determine the optimal tuning curve from parallel simulations of the more computationally intensive numerical model. This is an exhaustive iterative search which tests all possibilities to find the optimum, but, for ease of computation, a number of simplifying assumptions are made. First, it is assumed that the optimal tuning curve is identical for flood and ebb tides, and so an optimal curve is sought for only the first half of the tidal cycle and repeated thereafter. Second, it is assumed that the optimal dynamic tuning can be approximated by dividing the half tidal cycle into $M$ equal sub-cycles with fixed tuning. Third, it is assumed, following Vennell [14], from whose model the initial guesses are obtained, that the optimal turbine resistance only increases over the half tidal cycle.

Each sub-cycle is assigned $z=5$ choices for the value of the fixed tuning parameter (using $\alpha_{4}$ and $T S R$ rather than $k$, for simplicity), which are separated by tuning increments, $i$, of 0.1 for $\alpha_{4}$ and 0.5 for TSR (Fig. 4). The optimisa- 
(a)

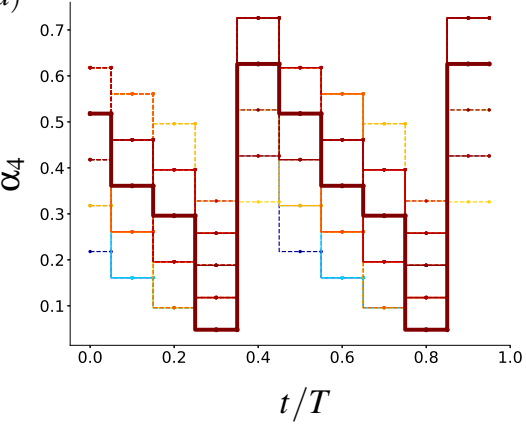

(b)

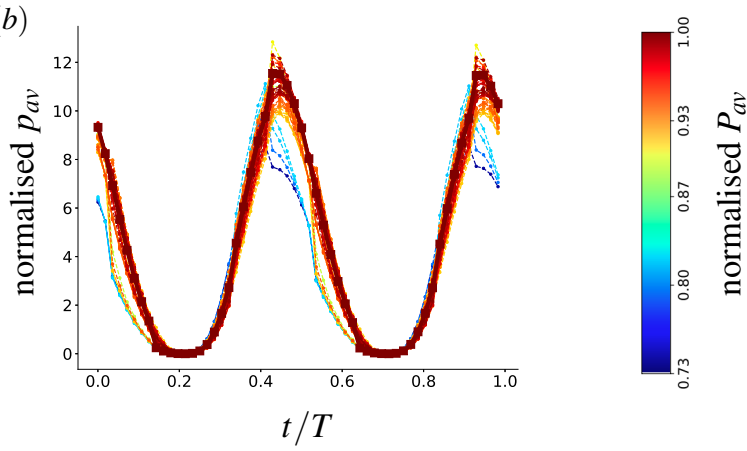

Fig. 4: Example variations in: (a) wake velocity coefficient, $\alpha_{4}$, and; $(b)$ corresponding instantaneous available power, $p_{a v}$, for a full-width array of actuator discs in time, $t$, over the tidal cycle, $T$. The curves are coloured according to the corresponding time-averaged available power, $P_{a v}$. (Colour online.)

tion scheme is found to be relatively insensitive to the choice of $i$ and $M$ (increasing $M$ above 5, for instance, is found to produce $<2 \%$ more power), and is adjusted and repeated if the optimal tuning curve is found to be limited by the $z$ initial choices for the fixed tuning parameter. Dynamic tuning strategies are also known to manipulate the phase of the oscillating currents $[8,13]$, and so the model is then rerun with all of the candidate tuning curves shifted forwards and backwards in time in 15-minute increments. Finally, after testing hundreds of candidate curves for each turbine arrangement, the optimal tuning curve is selected as that which produces the maximum time-averaged available power (Fig. 4).

\section{Local blockage}

The analytical and numerical models are now used to explore the potential for local blockage effects to enhance the performance of arrays comprising $N=1$ and 5 rows of actuator discs and tidal rotors operating with temporally fixed tuning in steady and oscillatory channel flow. A constant global blockage of $B_{G}=0.1$ is specified, and for each $N$, simulations are performed using eight different combinations of local blockage, $B_{L}$, and array blockage, $B_{A}$. Beginning with a full-width array $\left(B_{L}=0.1, B_{A}=1\right), B_{L}$ is increased (and $B_{A}$ reduced so that $B_{G}=B_{A} B_{L}$ remains constant) to a value of $B_{L}=0.3125\left(B_{A}=0.32\right)$ to create shorter and more highly blocked arrays. For each turbine arrangement, the value of the resistance coefficient, $k$, is held constant in time and tuned to obtain the maximum time-averaged available power, $P_{a v(\max )}$. Array performance is measured in terms of $P_{a v(\max )}$ for oscillatory flow, and for steady flow in terms of the maximum time-averaged global power coefficient, $C_{P G(\max )}$, which is defined here as the ratio of maximum time-averaged available power, $P_{a v(\max )}$, to the timeand cross-stream averaged channel-scale kinetic energy flux. The two-scale theory is then modified to include multiple rows of turbines and the tidal rotor model, and the theoretical $C_{P G(\max )}$ is used to estimate $P_{a v(\max )}$ and compare with the numerical results.

\subsection{Steady flow}

Figures $5(a)$ and $5(b)$ show the variations in $C_{P G(\max )}$ with $B_{L}$ for $N=1$ and 5 rows of discs and rotors operating with temporally fixed tuning in steady flow. The figures show excellent agreement between the analytical and numerical models for both turbine representations and numbers of rows. Larger discrepancies are apparent for both small and large values of $B_{L}$, which are most likely due to the different assumptions underlying the models, but, as noted in previous studies $[8,39]$, the two-scale theory is found to predict well the performance of partial-width arrays in steady flow.

Although the idealised disc tends to overestimate the performance of the more realistic rotor, the models are shown to respond similarly to increasing $B_{L}$ : for this channel, global blockage, and range of local blockages, $C_{P G(\max )}$ increases with increasing $B_{L}$ for $N=1$ and decreases with increasing $B_{L}$ for $N=5$. This finding shows that, consistent with existing work $[19,20,40]$, the effects of increasing local blockage vary with the number of turbine rows. To understand these $C_{P G(\max )}$ trends, it is useful to consider the corresponding variations in optimal resistance coefficient, $k_{(o p t)}$. For $N=1$, $k_{(o p t)}$ increases with increasing $B_{L}$ as the greater confinement of flow enables higher optimal thrusts, but for $N=5$, $k_{(o p t)}$ decreases with increasing $B_{L}$ so as to compensate for the much higher array resistance (Fig. 5(c)).

Figures 5(a) and 5(b) also show that, as compared to the discs, the performance of the rotors is less sensitive to the effects of local blockage. This too may be explained by considering the corresponding $k_{(o p t)}$ variations: for both $N=1$ and $5, k_{(o p t)}$ is shown to undergo less variation with $B_{L}$ for the rotor than for the disc (Fig. 5(c)). This is because for the rotor, the peak $C_{P}$ coincides with lower values of $k$ and $C_{T}$, and so, as compared to the disc, the amount by which $C_{P G(\max )}$ can be enhanced as the optimal array thrust increases is limited. For an isolated turbine, for instance, increasing $B$ from 0.1 to 0.3 results in a $\sim 66 \%$ increase in $C_{P(\max )}$ for the disc but only a $\sim 39 \%$ increase for the rotor (Table 1 ). Conversely, when the optimal array thrust reduces, the reduction in $k_{(o p t)}$ is lower for the rotor than the disc because it presents a lower resistance and thus requires a smaller tuning correction. For $N=5$, for instance, increasing $B_{L}$ from 0.1 to 0.3 results in an $\sim 18 \%$ decrease in $k_{(o p t)}$ for the disc but only a $\sim 5 \%$ de- 

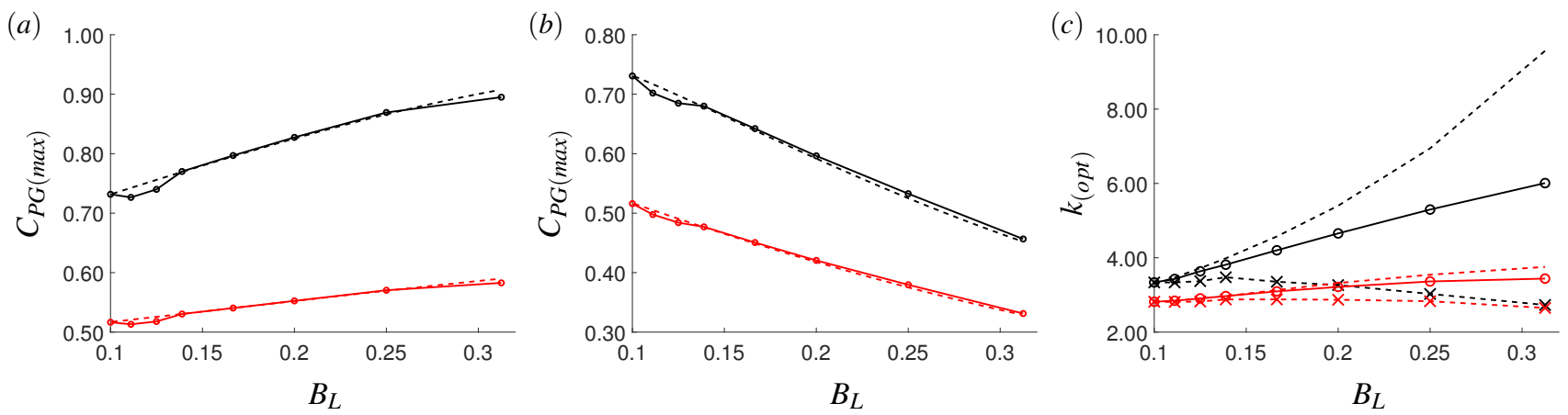

Fig. 5: Numerically (solid lines) and analytically (dashed lines) predicted variations in maximum time-averaged global power coefficient, $C_{P G(\max )}$, with local blockage, $B_{L}$, for: $(a)$ one, and; $(b)$ five rows of actuator discs (black) and tidal rotors (red) operating with temporally fixed tuning in steady flow, and; $(c)$ numerically predicted optimal resistance coefficients, $k_{(o p t)}$, for one (circles) and five (crosses) rows of turbines, and isolated turbines (dashed lines). (Colour online.)
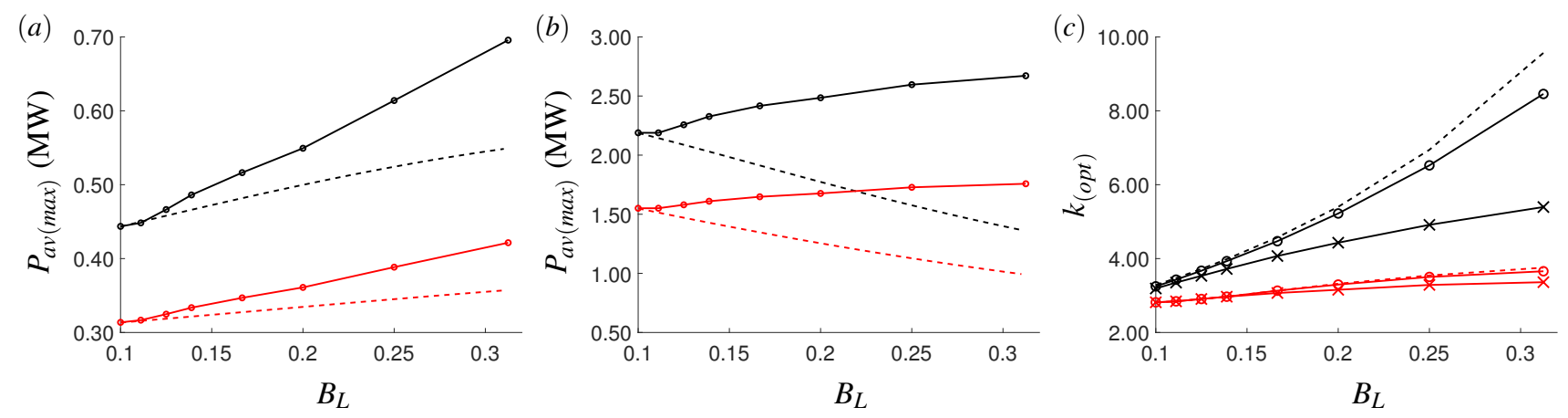

Fig. 6: Numerically (solid lines) and analytically (dashed lines) predicted variations in maximum time-averaged available power, $P_{a v(\max )}$, with local blockage, $B_{L}$, for: $(a)$ one, and; $(b)$ five rows of actuator discs (black) and tidal rotors (red) operating with temporally fixed tuning in oscillatory flow, and; $(c)$ numerically predicted optimal resistance coefficients, $k_{(o p t)}$, for one (circles) and five (crosses) rows of turbines, and isolated turbines (dashed lines). (Colour online.)

crease for the rotor.

Figure 5(c) shows that the difference in $k_{(o p t)}$ between the rotor and disc increases with increasing $B_{L}$, as the idealised disc generates a higher thrust, leading to greater power increases. Table 1 shows that as $B_{L}$ increases, so too does $k_{(o p t)}$ and the disc simply uses a larger $k$ and hence $C_{T}$ to produce a higher $C_{P(\max )}$. The rotor also uses a larger $k$ to maintain the optimal attack angle as the throughflow velocity increases, but $k_{(o p t)}$ increases more slowly with $B_{L}$ for the rotor than for the disc because the higher angular velocity reduces the angle of attack and hence lift-to-drag ratio. $C_{P(\max )}$ increases more quickly with $B_{L}$ for the idealised disc than the more realistic rotor, but the much higher $k_{(o p t)}$ and hence $C_{T(o p t)}$ results in a greater reduction in basin efficiency, $\eta_{(o p t)}$ (Table 1). By comparison, the decrease in $\eta_{(\text {opt })}$ is much smaller for the rotor because the corresponding increase in $k_{(o p t)}$ is much smaller. It should also be noted, however, that the present rotor is designed for $B_{L}=0.16$ and so should not be expected to perform optimally across the range of $B_{L}$ values considered here.

For $N=1$, the disc overestimates the performance of the rotor by a greater amount when $B_{L}$ is large, and by a lesser amount when $B_{L}$ is small, whilst the opposite is true for $N=5$. Again, this may be explained by considering the $k_{(o p t)}$ variations. For $N=1, k_{(o p t)}$ increases with increasing
$B_{L}$ because, for a single row of turbines, $C_{P G(\max )}$ may be increased by increasing the array thrust. For $N=5$, however, $k_{(o p t)}$ decreases with increasing $B_{L}$ because, for five rows of turbines, the applied thrust is sufficiently large that $C_{P G(\max )}$ can only be increased by applying a lower array thrust. The above finding is explained, then, by the realisation that when $k_{(o p t)}$ is small, the more realistic rotor is able to match more closely the performance of the idealised disc (Figs 2, 3).

Figure 5(c) also shows that, for this inertial channel, the $k_{(o p t)}$ variations which produce $C_{P G(\max )}$ for the partial-width arrays $\left(B_{L}>0.1\right)$ do not match those which produce $C_{P(\max )}$ for each turbine in isolation. This demonstrates the importance of tuning for turbines in arrays, because tuning each turbine as if it were in isolation would mean a significantly higher combined thrust, more flow being diverted around the array, and less power being produced as a result [18].

\subsection{Oscillatory flow}

Figures $6(a)$ and $6(b)$ show the variations in $P_{a v(\max )}$ with $B_{L}$ for $N=1$ and 5 rows of discs and rotors operating with temporally fixed tuning in oscillatory flow. In this case, it is clear that the two-scale theory is less well able to predict well the results of the numerical model. The figures show that in this inertial channel $\left(\lambda_{0}=0.2\right)$, the partial-width arrays $\left(B_{L}>0.1\right)$ produce considerably more power than the 
equivalent full-width arrays $\left(B_{L}=0.1\right)$. Moreover, the trends suggest that turbine arrangements with higher $B_{L}$ than those considered here can produce even more power. These findings are consistent with the work of Bonar et al. [8], who provide a more detailed description of the two-dimensional array-scale dynamics underlying this significant increase in power production. The departure from theory is most noticeable for $N=5$, where two-scale theory predicts that $P_{a v(\max )}$ should decrease with increasing local blockage but the numerical model shows $P_{a v(\max )}$ to increase.

For steady flow, Nishino and Willden [2] showed that the partial-width array divides the channel-scale flow into arrayscale core and bypass flows. For oscillatory flow, Bonar et al. [8] found that these array-scale flows oscillate side-byside but with their peak velocities separated by a phase difference. Using a two-dimensional numerical model, Bonar et al. [8] then showed that this phase difference results in the cross-stream diversion of array-scale bypass flow through the array each time the flow changes direction, and that under certain inertial oscillatory flow conditions, this 'reversal boost' mechanism can enhance considerably the power available to short and highly blocked turbine arrays. Naturally, however, this two-dimensional phenomenon cannot be captured using the existing one-dimensional theories, which explains the large differences in power observed for large $B_{L}$.

The effects of the reversal boost and hence departure from two-scale theory will, of course, be channel-specific, and have been amplified here by the selection of a channel with a naturally low ratio of drag to inertial forces. It is also worth noting, as Bonar et al. [8] do, that although the timeaveraged available power results may be relatively insensitive to variations in the horizontally and spatially averaged horizontal eddy viscosity coefficient, the magnitude of the observed reversal boost is expected to have been enhanced by the use of this model, which provides only a simplistic description of array-scale mixing processes.

Whereas in steady flow, the thrust from the turbines should be carefully calibrated so as to slow but not choke the flow through the array, in oscillatory flow, it may be more beneficial to apply a larger thrust so as to maximise the effects of these two-dimensional dynamics [8]. Figures $6(a)$ and $6(b)$ show that, for this channel, the benefits of this turbine arrangement and tuning strategy apply not only to a single row of turbines but also to five rows, and not only to discs but also to rotors. Comparing between the two different models, it is clear that the discs again overestimate the amount of power available to the rotors, but that the performance of the more realistic array can also be enhanced considerably by arranging and tuning the turbines to maximise the effect of the reversal boost. Increasing $B_{L}$ from 0.1 to 0.3125 , for instance, provides $\sim 10 \%$ more power for the rotor (as compared to $\sim 20 \%$ more for the disc) for $N=5$, and almost $\sim 35 \%$ more power (as compared to almost $\sim 60 \%$ more for the disc) for $N=1$. It should also be noted, however, that this performance enhancement requires the turbines to exert and withstand large thrusts, which will also affect their operational lifetime and hence the overall return on investment.

Figure $6(c)$ shows that the considerable increases in power at large $B_{L}$ are obtained by tuning the discs and rotors to apply much higher values of $k_{(o p t)}$ for $N=1$, but slightly lower values of $k_{(o p t)}$ for $N=5$, so as to compensate for the much higher array resistance. In steady flow, where the effects of array-scale diversion are detrimental to power production, Fig. 5(c) showed that, for steady flow, the optimal tunings for the arrays can be quite different to those for the isolated turbines. In oscillatory flow, however, Fig. 6(c) shows that the two sets of optimal tunings align more closely, because the available power can be enhanced by tuning the turbines to present a higher resistance to the incoming flow so as to maximise the effect of the reversal boost [8]. As in the case of steady flow, $k_{(o p t)}$ is shown to undergo less variation with $B_{L}$ for the more realistic rotor than the idealised disc. Unlike for steady flow, however, the disc is found to overestimate the rotor performance by a greater amount when $B_{L}$ is large, for both $N=1$ and 5 . This is because the disc is able to apply considerably more thrust and produce, by means of this reversal boost, considerably more power.

\section{Dynamic tuning}

Numerical methods are now used to explore the potential for dynamic tuning strategies to enhance the performance of arrays comprising $N=3,6$, and 10 rows of actuator discs and tidal rotors operating with temporally varying tuning in oscillatory channel flow. A constant global blockage of $B_{G}=0.1$ is again specified, and simulations are performed using five different combinations of local blockage, $B_{L}$, array blockage, $B_{A}$, and numbers of turbine rows, $N$. Beginning with $N=10$ full-width rows, $B_{L}$ is first increased from $B_{L}=0.1\left(B_{A}=1\right)$ to $B_{L}=1 / 3\left(B_{A}=0.3\right)$ to create shorter and more highly blocked arrays. Then, starting with the shortest and most highly blocked array $\left(B_{L}=1 / 3, B_{A}=0.3\right)$, $B_{L}$ is held constant as $N$ is reduced (and $B_{A}$ increased so that $N B_{G}=N B_{A} B_{L}$ remains constant) from 10 partial-width rows to 3 full-width rows. For each turbine arrangement, the maximum time-averaged available power, $P_{a v(\max )}$, is first sought using a temporally fixed turbine tuning and then, via the generate-and-test approach described above, the optimal dynamic tuning curve.

For the initial full-width array $\left(N=10, B_{L}=0.1, B_{A}=\right.$ $1)$, dynamic tuning is shown to increase $P_{a v(\max )}$, but only by $\sim 0.2 \%$ for the disc and $\sim 0.1 \%$ for the rotor (Figs $7(a)$, $7(b))$. Such small increases in $P_{a v(\max )}$ are to be expected in this case, however, because although this channel has a naturally low ratio of drag to inertial forces, $\lambda_{0}=0.2$ [7], the values of $B_{L}$ and the non-dimensional number of turbine rows, and hence the amount of turbine drag, are also quite small [14]. For these full-width arrays of discs and rotors (and others, for which the results are not shown), the optimal dynamic tuning curves and resulting increases in $P_{a v(\max )}$ are found to match very well with the theoretical predictions [14].

For this channel, increasing $B_{L}$ from 0.1 to $1 / 3$ is shown to first increase and then decrease $P_{a v(\max )}$ for both discs and rotors (Figs $7(a), 7(b)$ ), which suggests that the optimal lo- 

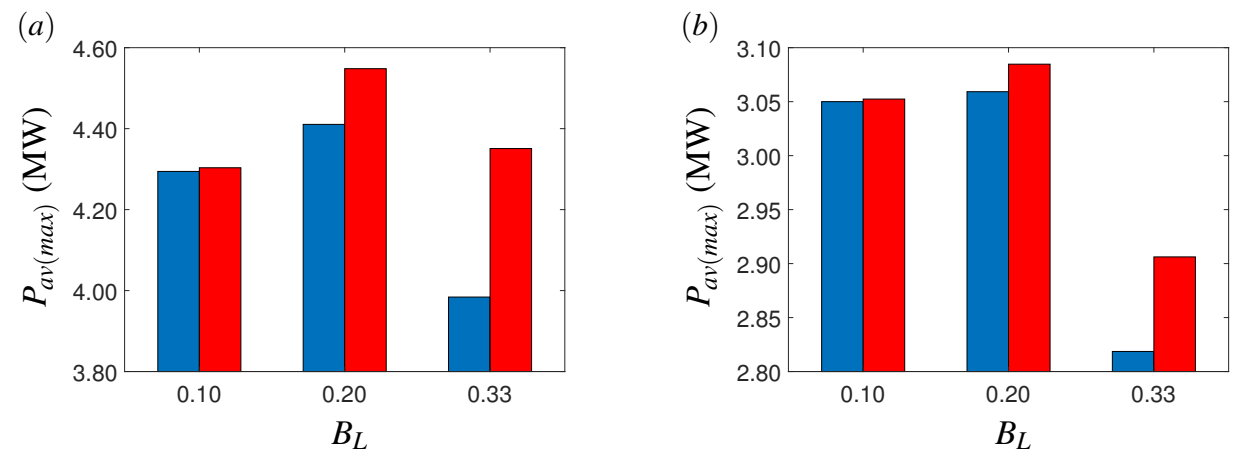

Fig. 7: Variations in maximum time-averaged available power, $P_{a v(\max )}$, with local blockage, $B_{L}$, for $N=10$ turbine rows, for: (a) actuator discs, and; (b) tidal rotors, operating in oscillatory flow with fixed (blue bars) and dynamic (red bars) tuning. (Colour online.)
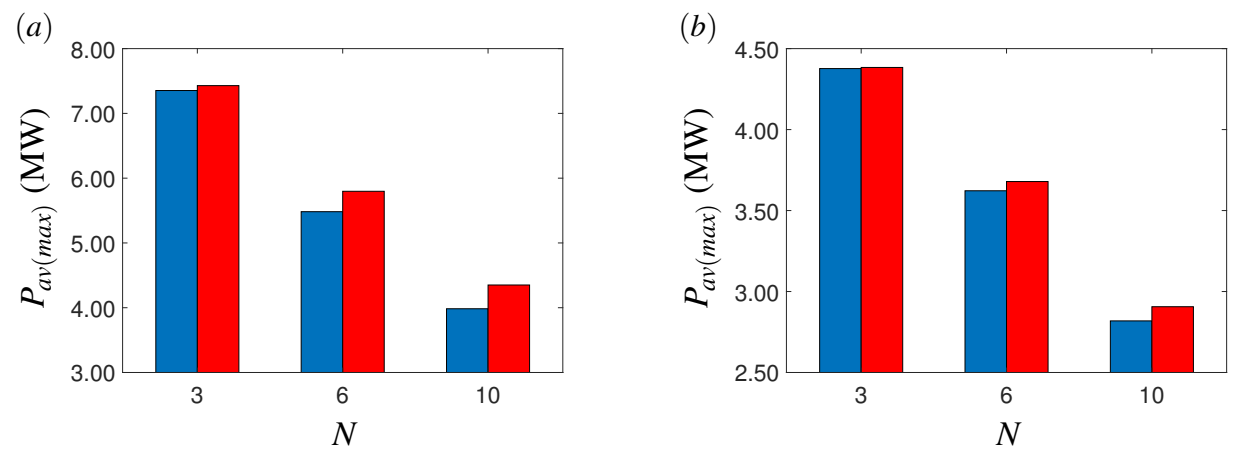

Fig. 8: Variations in maximum time-averaged available power, $P_{a v(\max )}$, with number of turbine rows, $N$, for local blockage, $B_{L}=1 / 3$, for: $(a)$ actuator discs, and; $(b)$ tidal rotors, operating in oscillatory flow with fixed (blue bars) and dynamic (red bars) tuning. (Colour online.)

cal blockage (for both turbine models and tuning strategies) lies between these two $B_{L}$ values [2]. $P_{a v(\max )}$ does not simply increase with $B_{L}$ because, in this case, $N$ is sufficiently large that the detrimental effects of turbine resistance on the channel flow rate outweigh the benefits of the reversal boost. This array-scale choking effect also explains why, as is consistent with theory [18], $P_{a v(\max )}$ is shown to decrease with increasing $N$ for both discs and rotors (Figs $8(a), 8(b)$ ).

Figures 7 and 8 also show that the amount by which dynamic tuning can enhance $P_{a v(\max )}$ increases both with $B_{L}$ for a given $N$, and with $N$ for a given $B_{L}$. These results are consistent with previous works on turbine tuning, which have shown that the importance of tuning increases with the potential for turbines to exert thrust on the incoming flow and hence affect their own performance $[15,18]$. The results are also consistent with present understanding of array performance: as $B_{L}$ increases (and $B_{A}$ reduces) for a given $N$ (or as $N$ increases and $B_{A}$ reduces for a given $B_{L}$ ), an array-scale bypass flow is introduced and a reversal boost dynamic is established [8]. Comparing the optimal dynamic tuning curves between full- and partial-width arrays (not shown) reveals significant differences designed to further enhance the reversal boost for partial-width arrays.

As with local blockage, the effects of dynamic tuning are consistent between the turbine models, though much less significant for the more realistic rotors than the idealised discs. The available power enhancement is greatest in both absolute and relative terms for $N=10$ and $B_{L}=1 / 3$, where dynamic tuning provides $\sim 9.2 \%$ more power for the discs but only $\sim 3.3 \%$ more power for the rotors. The benefits of dynamic tuning are shown to be much less significant, however, for both fewer turbine rows and lower local blockages.

\section{Conclusions}

Numerical simulations are used to explore the potential for local blockage effects and dynamic tuning strategies to enhance the performance of turbines in tidal channels. Full- and partial-width arrays of turbines, modelled using the volume-flux-constrained actuator disc and blade element momentum theories, are embedded within a twodimensional tidal channel. The channel is assigned a naturally low ratio of drag to inertial forces, and large numbers of turbine rows, $N$, and local blockage ratios, $B_{L}$, are used to amplify the effects of local blockage and dynamic tuning. To compare with the numerical results, the two-scale actuator disc theory of Nishino and Willden [2] is extended to incorporate tidal rotors, multiple rows of turbines, and coupled with an idealised model of channel-scale dynamics, and the theoretical dynamic tuning model of Vennell [14] is also extended to incorporate the tidal rotor representation.

The first set of findings concern the effects of local blockage on the performance of turbine arrays operating with temporally fixed tuning. For steady flow, the two-scale the- 
ory is shown to predict very well the results of the twodimensional numerical model. For oscillatory flow, however, numerical results show that the shorter and more highly blocked arrays produce considerably more power than predicted by the one-dimensional two-scale theory. These results support the findings of Bonar et al. [8], who showed that under certain oscillatory flow conditions, the power produced by a partial-width tidal turbine array can be much greater than predicted by two-scale theory. The departure from theory is evident for both actuator discs and tidal rotors, and most noticeable for $N=5$, where the two-scale theory predicts that the time-averaged available power, $P_{a v(\max )}$, should decrease with increasing $B_{L}$ but the numerical model shows $P_{a v(\max )}$ to increase.

The second set of findings concern the effects of dynamic tuning on tidal turbine performance. In all cases considered, the optimal dynamic tuning is shown to produce a greater $P_{a v(\max )}$ than a temporally fixed tuning. In most cases, however, these increases are quite small: even for this inertial channel, significant power increases through dynamic tuning are obtained only for large numbers of turbine rows or large local blockage ratios.

The observed trends are consistent between the two turbine models, but the effects of local blockage and dynamic tuning are found to be much less significant for the rotor than the disc. In essence, this is because a full exploitation of these power maximisation strategies requires very large array resistances, and, as compared to the idealised disc, the more realistic rotor has a limited capacity to exert thrust on the incoming flow. The rotor also experiences additional energy losses, such as blade tip losses, which the disc does not experience and which make it less suitable for operation under high-thrust conditions. In this case, however, realistic representation of rotor performance is also limited by the lack of a control strategy to maintain peak performance above the rated flow speed [26].

Although the present results suggest that the benefits of dynamic tuning will be limited, particularly for the more realistic rotors, there are still many aspects of tidal turbine and array performance to explore. One topic of interest would be to examine further the ways in which dynamic tuning can enhance the performance of short and highly blocked arrays operating in oscillatory channel flow. Another would be to consider the effects of varying the local blockage and/or resistance between subsequent rows of turbines: Vennell [41] considered the effect of variations in tuning between subsequent rows but not blockage; whilst Bonar et al. [42] examined the effect of variations in blockage and resistance across a single row but not between subsequent rows. These variations between rows would be particularly interesting when viewed in the context of maintaining optimal performance for an array to which new turbines are being added.

\section{Acknowledgements}

The authors wish to thank Bowen Cao for providing invaluable assistance in the preparation of this article. P.A.J.B. gratefully acknowledges support from Engi- neering and Physical Research Council (EPSRC) and Natural Environment Research Council (NERC) grant number EP/R007632/1.

\section{References}

[1] Vennell, R., Funke, S.W., Draper, S., Stevens, C., and Divett, T., 2015. "Designing large arrays of tidal turbines: A synthesis and review". Renew. Sust. Energy Rev., 41, pp. 454-472.

[2] Nishino, T. and Willden, R.H.J., 2012. "The efficiency of an array of tidal turbines partially blocking a wide channel". $J$. Fluid Mech., 708, pp. 596-606.

[3] Garrett, C. and Cummins, P., 2007. "The efficiency of a turbine in a tidal channel". J. Fluid Mech., 588, pp. 243-251.

[4] Schluntz, J. and Willden, R.H.J., 2015. "The effect of blockage on tidal turbine rotor design and performance". Renew. Energy, 81, pp. 432-441.

[5] Bahaj, A.S., Molland, A.F., Chaplin, J.R., and Batten, W.M.J., 2007. "Power and thrust measurements of marine current turbines under various hydrodynamic flow conditions in a cavitation tunnel and a towing tank". Renew. Energy, 32, pp. 407426.

[6] Vogel, C.R., Houlsby, G.T., and Willden, R.H.J., 2016. "Effect of free surface deformation on the extractable power of a finite width turbine array". Renew. Energy, 88, pp. 317-324.

[7] Garrett, C. and Cummins, P., 2005. "The power potential of tidal currents in channels". Proc. R. Soc. A, 461(2060), pp. 2563-2572.

[8] Bonar, P.A.J., Chen, L., Schnabl, A.M., Venugopal, V., Borthwick, A.G.L., and Adcock, T.A.A., 2019. "On the arrangement of tidal turbines in rough and oscillatory channel flow". J. Fluid Mech., 865, pp. 790-810.

[9] Vogel, C.R., Willden, R.H.J., and Houlsby, G.T., 2018. "Blade element momentum theory for a tidal turbine". Ocean Engng, 169, pp. 215-226.

[10] Adcock, T.A.A., Draper, S., Houlsby, G.T., Borthwick, A.G.L., and Serhadlioğlu, S., 2013. "The available power from tidal stream turbines in the Pentland Firth". Proc. $R$. Soc. A, 469(2157), p. 20130072.

[11] Serhadlığlu, S., Adcock, T.A.A., Houlsby, G.T., Draper, S., and Borthwick, A.G.L., 2013. "Tidal stream energy resource assessment of the Anglesey Skerries”. Int. J. Mar. Energy, 3, pp. e98-e111.

[12] Bonar, P.A.J., Schnabl, A.M., Lee, W.-K., and Adcock, T.A.A., 2018. "Assessment of the Malaysian tidal stream energy resource using an upper bound approach". J. Ocean Engng Mar. Energy, 4(2), pp. 99-109.

[13] Vennell, R. and Adcock, T.A.A., 2014. "Energy storage inherent in large tidal turbine farms". Proc. R. Soc. A, 470(2166), p. 20130580.

[14] Vennell, R., 2016. "An optimal tuning strategy for tidal turbines”. Proc. R. Soc. A, 472(2195), p. 20160047.

[15] Chen, L., Bonar, P.A.J., Vogel, C.R., and Adcock, T.A.A., 2019. "A note on the tuning of turbines in tidal channels". J. Ocean Engng Mar. Energy, 5(1), pp. 85-98.

[16] Houlsby, G.T., Draper, S., and Oldfield, M.L.G., 2008. Application of linear momentum actuator disc theory to open channel flow. Tech. Rep. OUEL 2296/08, Department of Engineering Science, University of Oxford.

[17] Houlsby, G.T. and Vogel, C.R., 2016. "The power available to tidal turbines in an open channel flow". Proc. ICE - Energy, 170(1), pp. 12-21. 
[18] Vennell, R., 2010. "Tuning turbines in a tidal channel". $J$. Fluid Mech., 663, pp. 253-267.

[19] Draper, S. and Nishino, T., 2014. "Centred and staggered arrangements of tidal turbines”. J. Fluid Mech., 739, pp. 72-93.

[20] Draper, S. and Nishino, T., 2014. "Centred and staggered arrangements of tidal turbines - erratum”. J. Fluid Mech., 743, p. 636.

[21] Draper, S., Nishino, T., Adcock, T.A.A., and Taylor, P.H., 2016. "Performance of an ideal turbine in an inviscid shear flow". J. Fluid Mech., 796, pp. 86-112.

[22] Whelan, J.I., Graham, J.M.R., and Peiró, J., 2009. "A freesurface and blockage correction for tidal turbines". J. Fluid Mech., 624, pp. 281-291.

[23] Nishino, T. and Willden, R.H.J., 2012. "Effects of 3-D channel blockage and turbulent wake mixing on the limit of power extraction by tidal turbines". Int. J. Heat Fluid Flow, 37, pp. 123-135.

[24] Vogel, C., 2014. "Theoretical limits to tidal stream energy extraction". DPhil Thesis, University of Oxford, UK.

[25] Wimshurst, A., Vogel, C., and Willden, R., 2018. "Cavitation limits on tidal turbine performance". Ocean Engng, 152, pp. 223-233.

[26] Vogel, C.R., Willden, R.H.J., and Houlsby, G.T., 2019. "Tidal stream turbine power capping in a head-driven tidal channel". Renew. Energy, 136, pp. 491-499.

[27] Cao, B., Willden, R.H.J., and Vogel, C.R., 2018. "Effects of blockage and freestream turbulence intensity on tidal rotor design and performance". In Proc. 3rd International Conference on Renewable Energies Offshore, Lisbon, Portugal.

[28] Wimshurst, A. and Willden, R.H.J., 2016. "Computational analysis of blockage designed tidal turbine rotors". In Proc. 2nd International Conference on Renewable Energies Offshore, Lisbon, Portugal.

[29] Willden, R.H.J., Nishino, T., and Schluntz, J., 2014. "Tidal stream energy: Designing for blockage". In Proc. 3rd Oxford Tidal Energy Workshop, Oxford, UK.

[30] Gong, X., Li, Y., and Lin, Z., 2018. "Effects of blockage, arrangement, and channel dynamics on performance of turbines in a tidal array". J. Renew. Sust. Energy, 10(1), p. 014501.

[31] Kubatko, E.J., Westerink, J.J., and Dawson, C., 2006. " $h p$ discontinuous Galerkin methods for advection dominated problems in shallow water flow". Comp. Meth. Appl. Mech. Engng, 196(1-3), pp. 437-451.

[32] Kubatko, E.J., Bunya, S., Dawson, C., and Westerink, J.J., 2009. "Dynamic $p$-adaptive Runge-Kutta discontinuous Galerkin methods for the shallow water equations". Comp. Meth. Appl. Mech. Engng, 198(21-26), pp. 1766-1774.

[33] Draper, S., Houlsby, G.T., Oldfield, M.L.G., and Borthwick, A.G.L., 2010. "Modelling tidal energy extraction in a depthaveraged coastal domain". IET Renew. Power Gen., 4(6), pp. 545-554.

[34] Draper, S., Borthwick, A.G.L., and Houlsby, G.T., 2013. "Energy potential of a tidal fence deployed near a coastal headland". Phil. Trans. Roy. Soc. A, 371(1985), p. 20120176.

[35] Draper, S., Stallard, T., Stansby, P., Way, S., and Adcock, T., 2013. "Laboratory scale experiments and preliminary modelling to investigate basin scale tidal stream energy extraction". In Proc. 10th European Wave and Tidal Energy Conference, Aalborg, Denmark.

[36] Schnabl, A.M., Moreira, T.M., Wood, D., Kubatko, E.J., Houlsby, G.T., McAdam, R.A., and Adcock, T.A.A., 2019. "Implementation of tidal stream turbines and tidal barrage structures in DG-SWEM”. In Proc. ASME 38th International
Conference on Ocean, Offshore and Arctic Engineering, Glasgow, UK.

[37] Soulsby, R., 1997. Dynamics of marine sands: a manual for practical applications. Thomas Telford Publications.

[38] Adcock, T.A.A., 2012. "On the Garrett \& Cummins limit". In Proc. 1st Oxford Tidal Energy Workshop, Oxford, UK.

[39] Perez-Campos, E. and Nishino, T., 2015. "Numerical validation of the two-scale actuator disc theory for marine turbine arrays". In Proc. 11th European Wave and Tidal Energy Conference, Nantes, France.

[40] Divett, T., Vennell, R., and Stevens, C., 2014. "Channel scale optimisation of large tidal turbine arrays in packed rows using large eddy simulations with adaptive mesh". In Proc. 2nd Asian Wave and Tidal Energy Conference, Tokyo, Japan.

[41] Vennell, R., 2011. "Tuning tidal turbines in-concert to maximise farm efficiency". J. Fluid Mech., 671, pp. 587-604.

[42] Bonar, P.A.J., Adcock, T.A.A., Venugopal, V., and Borthwick, A.G.L., 2018. "Performance of non-uniform tidal turbine arrays in uniform flow". J. Ocean Engng Mar. Energy, 4(3), pp. 231-241. 